\title{
In Situ Tribology Study of Nanoprobe Sliding on Defective Crystalline Surface
}

\author{
Y. Liao and L. D. Marks
}

Department of Materials Science and Engineering, Northwestern University, 2220

Campus Drive, Evanston, Illinois 60208, USA

\begin{abstract}
Although the relative motion of two bodies in contact involves a number of forces across the interface, influencing the nature of sliding and wear, frictional forces play a central role in everyday experience as it was in ancient times, arising in the fields of physics, chemistry, geology, biology and engineering. The understanding and evaluation of friction, especially atomic-scale friction, remains a challenge. It is suggested that dislocation motion mainly contributes frictional force in dislocation-rich interface between two crystalline bodies. The friction here is studied by observing the motion of dislocations driven by sliding of the probe in combination with an applied load of a nanoprobe. In this study a Nanofactory AFM-TEM holder is used in TEM (transmission electron microscope) system, the results of in situ observation of dislocation motion during probe sliding is analyzed and, finally the frictional force during nanoprobe sliding is characterized.
\end{abstract}

\section{Reference:}

Liquid-like tribology of gold studied by in situ TEM, A. P. Merkle, L. D. Marks, Wear 265 (2008) 1864-1869. 


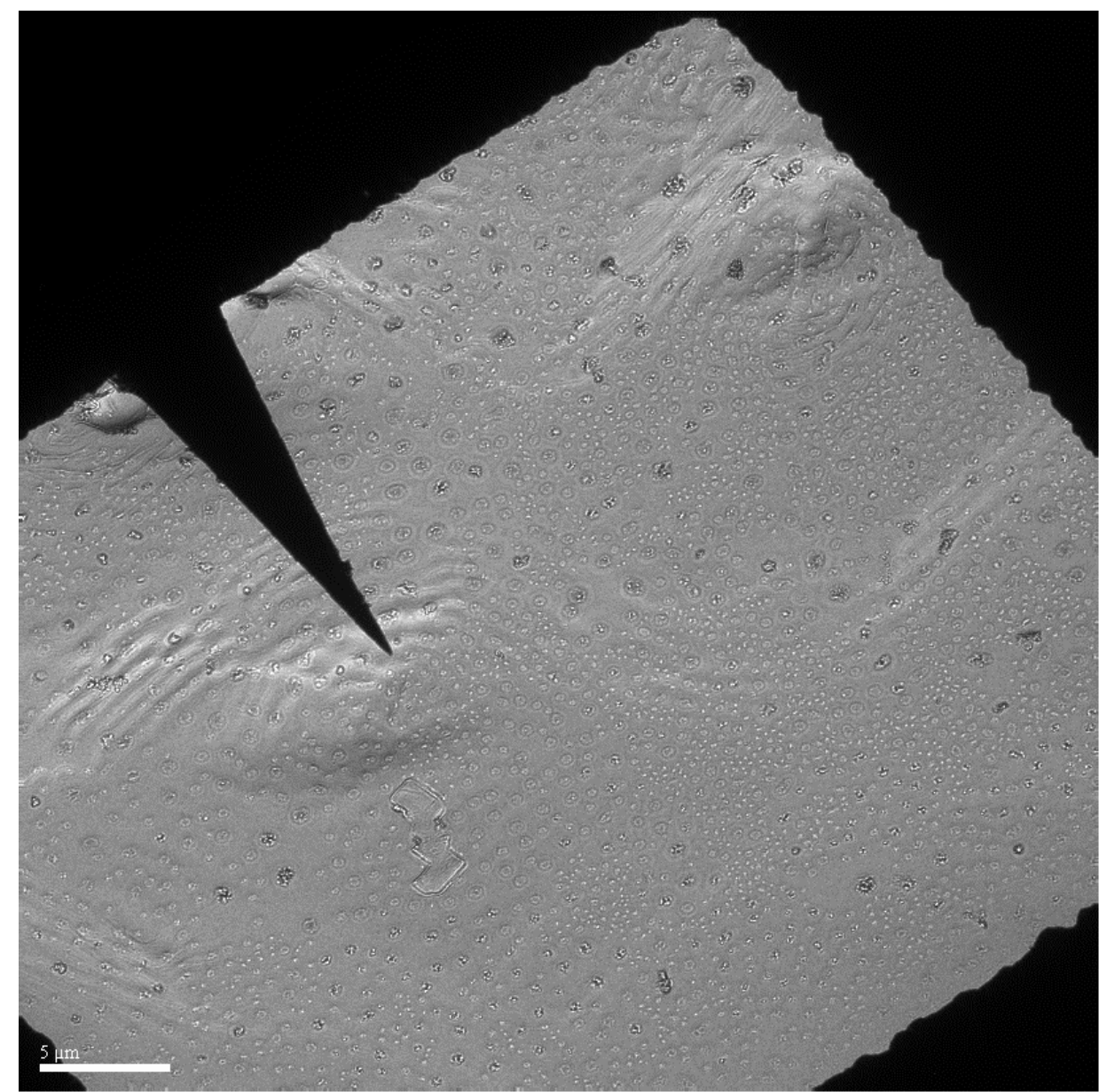

Figure 1. A nanoprobe sliding on a gold film with a thickness of $8 \mathrm{~nm}$. 